\title{
In situ Polymerisation of Pyrrole Within the Lattices of Mesoporous Hexagonal Silica Systems
}

\author{
Gaël Feraille, Peter J. S. Foot \& Richard J. Singer* \\ School of Pharmacy \& Chemistry, Faculty of Science, Engineering \& Computing, \\ Kingston University, Penrhyn Road, \\ Kingston upon Thames, Surrey, KT1 2EE, England.
}

\section{SUMMARY}

This study concerns the preparation and characterisation of electrically conducting polypyrrole-silica (PPy-Si) composites. Pyrrole monomers were adsorbed in nanoporous MCM-41 silicas exhibiting a hexagonal $\left(\mathrm{H}_{1}\right)$ geometry, some of which had been functionalised using aminopropyltriethoxysilane (APS), and chlorotrimethylsilane (TMS). Composites were then produced through oxidative polymerisation and doping with anions, mainly sodium sulphonates (methylbenzenesulphate (MBSA), dodecylbenzenesulphonate (DBSA) and diethylhexylsulphosuccinate (DEHS)). An attempt to produce similar composites via direct threading of "soluble" polypyrrole was also investigated in order to study the effect of confined geometries on the growth and properties of polypyrrole chains. The final composites exhibited semiconducting properties ( $\sigma$ up to $10^{-}$

${ }^{3} \mathrm{~S} . \mathrm{cm}^{-1}$ ), and improved thermal and environmental stabilities, making them suitable for applications such as sensors and antistatic agents.

Keywords: Polypyrroles (PPy), MCM-41, Oxidative polymerisation, in situ polymerisation

* Author for correspondence (r.singer@kingston.ac.uk)

\section{INTRODUCTION}

In principle, conducting polymers share the electrical properties of semiconductors or metals, with the mechanical properties of polymers. However, the $\pi$-electron system along the polymer backbone which confers rigidity and cross-linking points between the chains, makes simple polypyrroles amorphous, infusible, intractable and insoluble in common solvents. These and other drawbacks, such as lack of control over doping level and poor stability at high humidity, ${ }^{(1)}$ impose some limits on their exploitation. Over the years, researchers have tried to overcome these limitations via the formation of solutions, dispersions of the polymeric materials in their conducting forms, by copolymerisation with processable or substituted monomers and the preparation of conducting polypyrrole-based polymer blends. ${ }^{(2-6)}$ More recently, researchers have investigated the potential of in situ polymerisation, where guest entities, (a range of molecular species of different sizes and geometries), have been inserted into porous host solids, which provide a network of interconnected vacant lattice sites with free volume and appropriate geometry for the location and transport of the guest species. Bein and Enzel, ${ }^{(7-9)}$ and others, have concentrated their efforts on inorganic hosts, ${ }^{(10,11)}$ especially clays, zeolites, and porous oxides, whose large surface areas, high porosities, controllable and narrowly distributed pore sizes, make them very promising host candidates. Their resulting composites demonstrated that intercalated conducting polymers within such hosts possessed improved environmental stabilities and structural properties but with mostly insulating or poor semiconducting properties, due to very low polymer loadings.

In this study, our objective was to pursue the preparation of polypyrrole-silica composites with polypyrrole-rich inner surfaces, and semiconducting behaviour, via the in situ polymerisation of pyrrole monomers within the hexagonal lattices of MCM-41 materials. These materials, initially developed by Mobil in the nineties, exhibit 
high surface areas and adjustable pore diameters, and their structures are amorphous rather than crystalline at the nanometre scale, but are based overall on a specified geometry. ${ }^{(12,13)}$ These materials offer increased molecular diffusion rates in comparison with other silicates, and yet their channels are small enough to provide useful in-pore effects.

\section{EXPERIMENTAL}

\subsection{MCM-41 syntheses}

MCM-41 hosts were prepared via a mild hydrothermal non-ionic templating procedure, similar to that of Attard et $a l{ }^{(14)}$ Warm dilute $\mathrm{HCl}(0.5 \mathrm{M}$, Fisher) was added to polyoxyethylene $(\mathrm{n}=10)$ cetyl ether (Brij 56 , Aldrich) dissolved in warm (TEOS, Avocado). (Initial mole ratios TEOS/Brij 56/HCl: 8.03/1.00/2.07). Ethanol, the hydrolysis product of TEOS, was removed under gentle vacuum, since in large amounts it can disrupt the lyotropic behaviour of the surfactant phase. After $1 \mathrm{~h}$, the vessels were sealed to undertake final curing $\left(40^{\circ} \mathrm{C}, 7\right.$ days). Post synthesis, the template molecules were removed either by dissolution or calcination. Calcinations were conducted in air, using a Carbolite Muffle furnace. $\left(25-220^{\circ} \mathrm{C}, 5^{\circ} \mathrm{C} \cdot \mathrm{min}^{-1}\right.$ gradient; $5 \mathrm{~h}$ isotherm at $220^{\circ} \mathrm{C}$, then $220-550^{\circ} \mathrm{C}, 10^{\circ} \mathrm{C} \cdot \mathrm{min}^{-1}$ gradient; $10 \mathrm{~h}$ isothermal at $\left.550^{\circ} \mathrm{C}\right)$. Soxhlet extractions were conducted for $12 \mathrm{~h}$, using ethanol $(\mathrm{BDH}) /$ water $(90 \% \mathrm{v} / \mathrm{v})$ as the solvent.

Surface modification (silylation) of some samples was carried out using either chlorotrimethylsilane (TMS, Aldrich) or aminopropyltriethoxysilane (APS, Aldrich). MCM-41 was added to TMS/toluene $(0.6 \% \mathrm{v} / \mathrm{v}, \mathrm{BDH})$ mixtures and refluxed for $10 \mathrm{~h}$. The resulting powders were washed with acetone, and any side-products removed by soxhlet extractions, using dichloromethane/diethyl ether $(45 \% \mathrm{v} / \mathrm{v}, \mathrm{BDH})$ solvent. APS was hydrolysed in ethanol/water $(90 \% \mathrm{v} / \mathrm{v}, \mathrm{BDH})\left(25^{\circ} \mathrm{C}, 6 \mathrm{~h}\right) . \mathrm{MCM}-41$ was then stirred with the mixtures $\left(25^{\circ} \mathrm{C}\right.$, 12h). After filtration the resulting powders were washed with ethanol/water $(90 \% \mathrm{v} / \mathrm{v}, \mathrm{BDH})$ to remove any free APS. The washed powders were dried under vacuum $\left(40^{\circ} \mathrm{C}, 48 \mathrm{~h}\right)$, and stored in desiccators.

\subsection{Preparation of polypyrrole-silica composites, using in situ polymerisation or direct threading}

APS/TMS or unmodified MCM-41 were poured into pyrrole/pentane mixtures $\left(6 \% \mathrm{w} / \mathrm{v}\right.$, Avocado/BDH, $25^{\circ} \mathrm{C}$, $12 \mathrm{~h})$. These were filtered, washed with pentane, dried under vacuum and stored dry at room temperature. In situ polymerisation was achieved using different oxidant/dopant combinations (Table 1). Pyrrole-MCM-41 powders were added to aqueous solutions of NaDBSA, NaMBSA or NaDEHS, and the resulting mixtures were cooled to $0^{\circ} \mathrm{C}$. Aqueous solutions of iron (III) chloride (Aldrich) or ammonium persulphate (Aldrich) were slowly added and allowed to react for $10 \mathrm{~h}$. After filtration the resulting powders were washed with IMS/water (50\% v/v) and dried under vacuum $\left(40^{\circ} \mathrm{C}, 48 \mathrm{~h}\right)$. In order to improve threading, soluble polypyrroles (Table 2 ) were dissolved in DMSO (BDH), and the solutions were filtered to remove any undissolved polymer. APS/TMS or unmodified MCM-41 were stirred with the polymer solution $\left(100^{\circ} \mathrm{C}, 6 \mathrm{~h}\right)$. After filtration, the resulting powders were washed with DMSO and ethanol and dried under vacuum $\left(40^{\circ} \mathrm{C}, 48 \mathrm{~h}\right)$. The composites were stored in air or in moisture-free environments, either at ambient temperature or at $80^{\circ} \mathrm{C}$.

\subsection{Recovery of doped polypyrroles from host lattices.}

De-threading was conducted for $10 \mathrm{~h}$ in DMSO. Extraction cakes were filtered off and washed with several portions of warm DMSO. Extraction and filtration liquors were combined and the polymers were recovered by solvent evaporation. Hydrolyses of silica lattices were carried out in dilute $\mathrm{KOH}$ (5\% w/w, Aldrich) solution. The mixtures were filtered and the resulting materials were washed with water and ethanol, dried under vacuum $\left(40^{\circ} \mathrm{C}, 48 \mathrm{~h}\right)$ and stored in a desiccator. 


\begin{tabular}{|l|c|c|c|}
\hline \multicolumn{1}{|c|}{$\begin{array}{c}\text { Oxidant/ } \\
\text { Dopant }\end{array}$} & Oxidant & Dopant & $\mathbf{H}_{2} \mathbf{O}$ \\
\hline $\mathrm{FeCl}_{3} / \mathrm{MBSA}$ & 0.01 & 0.01 & 5.56 \\
$\mathrm{FeCl}_{3} / \mathrm{DBSA}$ & 0.01 & 0.01 & 5.56 \\
$\mathrm{FeCl}_{3} / \mathrm{DBSA}$ ex & 0.008 & 0.025 & 5.56 \\
$\mathrm{FeCl}_{3} / \mathrm{DEHS}$ & 0.008 & 0.015 & 5.56 \\
$\mathrm{FeCl}_{3}$ & 0.01 & 0.01 & 5.56 \\
$\left(\mathrm{NH}_{4}\right)_{2} \mathrm{~S}_{2} \mathrm{O}_{8} /$ & 0.005 & 0.01 & 5.56 \\
$\left.\mathrm{MBSA}_{(\mathrm{NH}}\right)_{2} \mathrm{~S}_{2} \mathrm{O}_{8} /$ & 0.005 & 0.01 & 5.56 \\
$\mathrm{DBSA}$ & & & \\
\hline
\end{tabular}

Table 1: $\quad$ In Situ polymerisation of pyrrole (initial molar ratios).

\begin{tabular}{|c|c|c|c|c|c|}
\hline Polypyrrole & Pyrrole & NaDBSA $_{\text {ex }}$ & NaDEHS & $\left(\mathrm{NH}_{4}\right) \mathrm{S}_{2} \mathrm{O}_{8}$ & $\mathrm{H}_{2} \mathrm{O}$ \\
\hline $\mathrm{PPy}\left(\mathrm{DBSA}_{\mathrm{ex}}\right)$ & 0.040 & 0.030 & --- & 0.008 & 5.56 \\
\hline PPy(DEHS) & 0.040 & --- & 0.015 & 0.008 & 5.56 \\
\hline
\end{tabular}

Table 2: SSoluble" polypyrrole syntheses (initial molar ratios).

\subsection{Characterisation}

Diffuse reflectance infrared Fourier-transform (DRIFT) spectroscopy was carried out at ambient temperature and pressure, on a Perkin-Elmer Spectrum One infrared spectrometer, using an external Graseby Specac Selector $^{\mathrm{TM}}$. Samples were dispersed in $\mathrm{KBr}$ (Fisher) and analysed as fine powders. Powder X-ray diffraction (XRD) was performed on a Bruker AXS generator, with a D8 Advance automatic goniometer controlled by Bruker XRD software. The XRD patterns were obtained with CuK $\alpha$ radiation $(0.154 \mathrm{~nm}, 40 \mathrm{kV}-30 \mathrm{~mA})$, using a nickel filter. Thermogravimetric analyses were carried out using a Mettler M3 furnace, combined with a TA processor TC 10A. Combustions were performed with 5, 10 and $20^{\circ} \mathrm{C} \cdot \mathrm{min}^{-1}$ gradients, between 50 and $650^{\circ} \mathrm{C}$, in air or nitrogen atmospheres. Gel permeation chromatography (GPC) analyses were performed on a Waters HPLC system (controlled by Millenium 32, 3.01), combined with Polymer Laboratories PLgel 5 $\mu$ m MIXED-C, 600x7.5 mm column and its Polymer Laboratories PLgel 5 $\mu \mathrm{m}$ GUARD, 50x7.5mm guard column. The analyses were performed at $70^{\circ} \mathrm{C}$, using a DMSO (HPLC grade) mobile phase.

Room temperature pentane adsorption was used to screen materials whose porosity had been compromised by template removal. Subsequently, nitrogen adsorption isotherms were determined gravimetrically at $77 \mathrm{~K}$ up to the saturated vapour pressure of the adsorbate by the addition or removal of aliquots of nitrogen. Calibrated quartz springs were used for mass determination. Specific surface areas were measured using a BET model, while the pore volumes and pore distributions were derived from the BJH method. Kjeldahl analyses were carried out to estimate the amount of nitrogen in intermediates and polymer composites. Particle size estimations were made on gold coated samples using a JEOL-6310 scanning electron microscope (SEM), operated at $15 \mathrm{kV}$ accelerating voltage, and combined with an Oxford Instruments ISIS $200 \mathrm{X}$-ray analysis system (fitted with an ultra-thin window $\mathrm{Si}(\mathrm{Li}) \mathrm{X}$-ray detector). The system was controlled by the ISIS 200 software, and operated using ZAF correction routines. The dc conductivity measurements were performed on compressed pellets $\left(75 \mathrm{~kg} . \mathrm{mm}^{2}\right.$ pressure, under vacuum), using a four-probe conductivity method (square Van der Pauw arrangement ${ }^{(15)}$ ). All measurements were performed using a Keithley 617 programmable electrometer and a Keithley 224 programmable current source. Temperature dependent conductivity measurements were recorded, using the four-probe method. A Linkam TMS 91 hot stage, fitted with a Linkam CS 196 cooling system, was used as heating source. $\mathrm{A} \pm 5^{\circ} \mathrm{C} \cdot \mathrm{min}^{-1}$ gradient was used in both heating and cooling phases, and the measurements were recorded every $25^{\circ} \mathrm{C}$. 


\section{RESULTS AND DISCUSSION}

In order to enhance the affinity of pyrrole monomers for the host lattice, it was necessary to functionalise the surfaces of the silica host materials. However, one has to take in account that the functionalisation of the inner surface of the host must interfere as little as possible with the mechanism of the polymerisation reaction, which, in the case of polypyrroles, implies that the pyrrole $\alpha$-carbons must remain free. The objective of silylation was, in the case of TMS, to dramatically increase the affinity between the host lattices and the pyrrole monomers. By creating a relatively hydrophobic environment, the intake of pyrrole should be greatly improved. In contrast, the purpose of the APS-silylation was to create $\mathrm{NH}_{2}$ "anchoring" sites, which should create electron donor-acceptor interactions with the pyrrole hydrogen attached to the nitrogen, which is slightly acidic.

\subsection{MCM-41 and their modified derivatives}

All MCM-41 materials were found to be composed of quasi-spherical particles of uniform size $(5-10 \mu \mathrm{m})$. Their XRD patterns exhibit an intense peak associated with the (100) reflection, if hexagonal geometry is assumed, and two other small diffractions indicative of (110) and (200) planes, which are characteristic of highly ordered hexagonal pore packing motifs. Template removal by solvent extraction resulted in MCM-41 exhibiting similar diffraction patterns to those of their templated precursors. Samples obtained by calcination tended to exhibit narrower patterns. Upon calcination, the (100) diffraction slightly increased in intensity, while their peak positions are shifted towards higher $2 \theta$ angles, indicating a slight shrinkage in pore size $(0.2-0.4 \mathrm{~nm})$ presumably due to condensation of silanol groups or structural changes brought about by annealing. Finally, structural parameters remained virtually unchanged upon functionalisation, which indicates that either silylation is not uniform or that silylating molecules do not adopt particular orientations when grafted to the MCM-41 surfaces. Nitrogen adsorption experiments indicated type IV isotherms as expected. Pore size determinations were characteristic of uniform pore systems with overall narrow size distributions. It should also be noted that, despite being relatively small, the shape of the hysteresis observed in MCM-41 (Figure 1), which can be considered "type A", is characteristic of cylindrical capillaries open at both ends, and thus hexagonal pore systems. ${ }^{(16)}$ In the case of silylated MCM-41, our main concern resided in the possible effects of silylating agents on the overall porosity of the hosts, indeed one could imagine that APS and TMS incorporations, should lead to decrease of surface areas and pore dimensions and, in the case of APS, could even block the entry of the pores. Indeed, APS molecules have a tendency to react with the first free-hydroxyl groups they encounter on the surface and at the entrances of the pores, and as demonstrated by Perruchot et al. ${ }^{(17)}$ this results in the formation of a layer that can progressively block these entrances.

\section{Figure 1: Nitrogen adsorption isotherms in (a) MCM-41/APS-MCM-41, (b) MCM-41/TMS-MCM-41 materials}

The adsorption isotherms of functionalised MCM-41 show no dramatic losses of porosity in comparison to unmodified samples which would be expected in the case of blocked pores, but rather small hysteresis with accentuated "type E" patterns (Figure 1). These loops reflect a slight inhomogeneous pore system, a mixture of cylindrical pores of different diameters with a slight neck effect at their entrances, which is also confirmed by the pore size distribution studies of these materials $(\varnothing \approx 2 \mathrm{~nm})$. We conclude from this that grafting takes place not only on the external surfaces of the materials, but also inside the pores. Further confirmation comes from DRIFT experiments. 


\begin{tabular}{|c|c|c|c|c|c|c|c|}
\hline MCM-41 & $\begin{array}{l}2 \theta \text { angles } \\
\text { (degrees) }\end{array}$ & $\begin{array}{c}\text { d- } \\
\text { spacing* } \\
(\mathrm{nm})\end{array}$ & $\begin{array}{c}\mathbf{a}_{\mathbf{o}} \\
(\mathbf{n m})\end{array}$ & $\begin{array}{l}\text { Surf. Area } \\
\left(\mathrm{m}^{2} \cdot \mathrm{g}^{-1}\right)\end{array}$ & $\begin{array}{c}\varnothing \\
(\mathbf{n m})\end{array}$ & $\begin{array}{c}\text { Pore } \\
\text { Vol. } \\
\left(\mathrm{cm}^{3} \cdot \mathrm{g}^{-1}\right)\end{array}$ & $\begin{array}{c}\mathbf{T} \\
(\mathbf{n m})\end{array}$ \\
\hline $\begin{array}{l}\text { Templated } \\
\text { Template-free }\end{array}$ & 2.49 & 3.55 & 4.10 & --- & --- & --- & --- \\
\hline Calcination & 2.50 & 3.52 & 4.07 & $1003( \pm 18)$ & 2.14 & 0.64 & 1.96 \\
\hline $\begin{array}{l}\text { Solvent } \\
\text { extraction }\end{array}$ & 2.49 & 3.55 & 4.10 & $1007( \pm 21)$ & 2.15 & 0.65 & $\begin{array}{l}1.96 \\
---\end{array}$ \\
\hline Silylated & 2.49 & 3.54 & 4.09 & $870( \pm$ & 1.81 & 0.55 & --- \\
\hline $\begin{array}{l}\text { TMS } \\
\text { APS }\end{array}$ & 2.55 & 3.46 & 3.99 & $\begin{array}{c}17) \\
734( \pm 19)\end{array}$ & 1.58 & 0.49 & --- \\
\hline
\end{tabular}

Table 3: Structure dimensions in MCM-41 materials.

MCM-41 materials exhibit similar features, due to framework silicate, to those found in other common silicate materials such as zeolites. (Water and $\mathrm{H}$-bonded silanol stretches at $3700-3000 \mathrm{~cm}^{-1}, \mathrm{H}_{2} \mathrm{O}$ bending modes at $1650-1600 \mathrm{~cm}^{-1}$, Si-O-Si stretches at $1100-1000 \mathrm{~cm}^{-1}$ and $\sim 800 \mathrm{~cm}^{-1}$ and Si-O-Si bending modes at $\sim 470 \mathrm{~cm}^{-1}$ ). However, the lower water content found in MCM-41 materials results in narrower bands around $3700-3000 \mathrm{~cm}^{-}$ , making the free-silanol absorption band visible $\left(3750 \mathrm{~cm}^{-1}\right)$. This is more obvious in freshly calcined samples, where the resolution between the two bands is greatly improved. Upon functionalisation, the intensity of the free-silanol band decreases, and in some cases the band disappears (Figure 2). This confirms that the APS- and TMS-silylations were successful and, that of the three different silanol species present on the silica surface, only the free silanols (single and geminal) are accessible to the silylating agent. Bands in the $\mathrm{C}-\mathrm{H}$ aliphatic stretching region $\left(3000-2900 \mathrm{~cm}^{-1}\right)$ are present in grafted silicas. In the case of APS-MCM-41, additional bands, at $\sim 1550$ and $1500 \mathrm{~cm}^{-1}$, are attributed to the $\mathrm{NH}_{2}$ and $\mathrm{NH}_{3}{ }^{+}$deformation of the "anchoring" sites. A minority of samples showed a weak absorption band at $3700 \mathrm{~cm}^{-1}$, which we attribute to a type of free-silanol derived from a geminal $\mathrm{OH}$ adjacent to a grafted TMS or APS group.

\section{Figure 2: DRIFT spectra of unmodified MCM-41/APS-MCM-41, MCM-41/TMS-MCM-41 materials.}

Thermogravimetric analyses show MCM-41 materials to have good thermal stability up to $650^{\circ} \mathrm{C}$; low mass losses resulting mainly from removal of residual water $\left(80^{\circ}-100^{\circ} \mathrm{C}\right)$ and progressive de-hydroxylation of silanols present on the surface. These losses are found to be much lower in functionalised materials than in unmodified analogues, especially in the case of TMS-MCM-41, where the loss of water is sometimes not detected. This phenomenon confirms that the hydrophilic behaviour of the silica surfaces has been dramatically altered upon functionalisation. The thermograms of silylated materials also exhibit large weight losses in the $300^{\circ} \mathrm{C}$ region, which corroborate our assumption of substantial APS and TMS functionalisations. By combining thermogravimetric and Kjeldahl analyses, we estimated the APS and TMS loadings to be $28 \%$ and $19 \%$ respectively.

All samples exhibited good environmental stabilities when kept in water-free environment. By contrast those stored in atmospheric conditions showed loss of overall nanoporosity over time. This is explained by a progressive collapse of the silicate structure, initiated by atmospheric moisture, which catalyses the hydrolysis of the Si-O-Si bonds; a phenomenon also reported by other teams, especially in the case of materials prepared from sodium metasilicate, where this hydrolysis is enhanced by the trapped alkali Na cations. Finally, as expected from materials long recognised to exhibit high resistivity, MCM-41, silylated or not, were found to be insulators $\left(<10^{-9} \mathrm{~S} . \mathrm{cm}^{-1}\right)$.

\subsection{Polypyrrole-silica composites produced by in situ polymerisation}

Polymer loadings of 3\% w/w in PPy-MCM-41 composites are far below that which MCM-41 is theoretically able to accommodate, thus corroborating our belief that the hydrophilic surfaces of the host do not facilitate the adsorption of pyrrole monomers. By contrast, composites produced within the lattices of TMS-MCM-41 exhibit 
increased polymeric loadings $(7 \%)$ despite the reduction in pore volume caused by silylation. The hydrophobicity introduced by TMS is apparently sufficient to attract larger amounts of monomer to allow the growth of polypyrrole chains. In APS-MCM-41, the polymer loading is still higher at $12 \%$. Our assumption is that the interaction of pyrrole through the $\mathrm{NH}_{2}$ groups of APS pendants is strong enough to retain the majority of the monomers sorbed, whereas in the case of TMS-MCM-41 weaker interactions allow substantial amounts of the monomeric material to diffuse away from the pores. The interaction between the pyrrole monomer and the amine groups is confirmed by the infrared studies of PPy-APS-MCM-41 composites, where the characteristic APS absorption band present in the $1550 \mathrm{~cm}^{-1}$ region tends to decrease in intensity or disappear when pyrrole monomers are introduced and increase on removal.

\section{Figure 3: DRIFT spectra of PPy-APS-MCM41 composites.}

A limiting factor for polymer growth is the confined geometry of the MCM-41 lattice. To understand its effect, intercalated polymers were retrieved by de-threading or lattice dissolution. De-threading was only partially successful, leaving substantial amounts of polymeric material within the host lattice, while lattice dissolution more effective. The MCM-41 lattice was found to dramatically affect the level of doping present in the recovered polypyrrole chains compared to that in conventional polypyrroles. A direct result of this decrease in polymer/dopant (P/D) ratios was a substantial loss of solubility. Solubility in polypyrroles is derived from the presence of large dopant anions such as DBSA and DEHS, which not only reduce the possibility of crosslinking, but also act as surfactants allowing the polypyrrole to be dispersed in selected solvents. The confined geometry also limits the mobility of polypyrrole chains within the silica lattices, which, combined with the decrease in solubility, explains why de-threading was ineffective, and resulted mainly in the retrieval of small and medium size polypyrrole chains. Longer chains are believed to remain in the host lattice. This partially explains why values of average molecular weight from materials recovered by de-threading are, smaller than those of materials recovered by lattice dissolution. Lattice dissolution allows the majority of the polymer to be recovered, giving a more accurate weight-average molecular weight $\left(\bar{M}_{w}\right)$.

\begin{tabular}{|c|c|c|c|c|}
\hline \multirow[b]{2}{*}{ Dopant anions } & \multicolumn{2}{|c|}{ Conventional polypyrroles } & \multicolumn{2}{|c|}{ Polypyrroles (In situ polymerisation) } \\
\hline & $\begin{array}{c}\text { Dopant loading } \\
(\mathrm{w} / \mathrm{w} \%)\end{array}$ & $\begin{array}{c}\text { Average repeating } \\
\text { unit }\end{array}$ & $\begin{array}{c}\text { Dopant loading } \\
(\mathrm{w} / \mathrm{w} \%)\end{array}$ & $\begin{array}{c}\text { Average repeating } \\
\text { unit }\end{array}$ \\
\hline DBSA & $40-45$ & {$\left[(\mathrm{Py})_{6}{ }^{+}(\mathrm{DBSA})^{-}\right]$} & $\sim 30$ & {$\left[(\mathrm{Py})_{11}{ }^{+}(\mathrm{DBSA})^{-}\right]$} \\
\hline $\mathrm{DBSA}_{\mathrm{ex}}$ & 55 & {$\left[\left(\mathrm{Py}_{4}{ }^{+}(\mathrm{DBSA})^{-}\right]\right.$} & $\sim 30$ & {$\left[(\mathrm{Py})_{11}+(\mathrm{DBSA})^{-}\right]$} \\
\hline MBSA & $36-41$ & {$\left[\left(\mathrm{Py}_{4}{ }^{+}(\mathrm{MBSA})^{-}\right]\right.$} & $\sim 30$ & {$\left[\left(\mathrm{Py}_{6}{ }^{+}(\mathrm{MBSA})^{-}\right]\right.$} \\
\hline DEHS & $50-54$ & {$\left[(\mathrm{Py})_{5}^{+}(\mathrm{DEHS})^{-}\right]$} & $\sim 45$ & {$\left[(\mathrm{Py})_{8}^{+}(\mathrm{DEHS})^{-}\right]$} \\
\hline
\end{tabular}

Table 4: $\quad$ Dopant loading and average repeating unit of polypyrroles synthesised in solution or by in situ polymerisation

PPy-APS-MCM-41 composites, which were found to contain the highest polypyrrole contents, exhibited the highest weight-average molecular weights. Their estimated polypyrrole chain lengths derived from average molecular weights, assuming that 1 mole of DEHS doped 8 moles of pyrrole units $\left(\mathrm{M}_{\mathrm{W}}=971.74 \mathrm{~g} \mathrm{~mol}^{-1}\right)$, represented $19\left[(\mathrm{Py})_{5}{ }^{+}(\mathrm{DEHS})^{-}\right]$units and, consequently, 152 pyrrole units.. These values are much smaller than those typically observed in conventional polypyrroles, ${ }^{(18)}$ confirming that the confined geometry of MCM-41 restricts the growth of polypyrrole chains.

\begin{tabular}{|c|c|c|}
\hline PPy(DEHS) & Lattice dissolution & De-threading \\
\hline PPy-MCM-41 & $<2000$ & $<2000$ \\
PPy-APS-MCM-41 & 18,502 & 13,604 \\
PPy-TMS-MCM-41 & 14,576 & 10,689 \\
\hline
\end{tabular}

Table 5: Weight-average molecular weights $\left(\bar{M}_{w} /\right.$ g.mol $\left.^{-1}\right)$ in PPy(DEHS) produced by in situ polymerisation 
As expected, the dc conductivities recorded in PPy(MBSA)-MCM- $41^{\mathrm{a}}$ composites are generally much lower than those found in conventional polypyrroles, and higher than those in composites doped with other sulphonate anions. However, unlike the case of conventional polypyrroles, these differences in dc conductivity are not simply connected to the nature of the dopant but also to the effect of the geometry of the host on these dopants. As seen earlier, the lattice limits the number of dopants present along the chain, especially of larger dopant anions; this is confirmed by the estimated numbers of repeating units, where (P/D) ratios in PPy(MBSA) were found to be much lower than in PPy(DBSA) or PPy(DEHS). The dopants are the source of the generous supply of potential charge carriers, which contribute to dc conductivity. A reduction in their concentration results in the reduction of the concentration of carriers and thus limit the dc conductivity of the composites. Overall, the conductivity is found to be several orders of magnitude lower than those found in conventional polypyrrole analogues. However, dc conductivity is not only largely dependent on the carrier concentrations, but also on their mobility along the chain, which should not be greatly affected by such intricate geometry. Indeed, as seen earlier, the hexagonal geometries of MCM-41 allow the growth of polypyrrole chains within their unidirectional pores, and so ensure a continuous conducting path. One could also predict that 2-D hopping should be favoured by the lack of free space within the pores, which forces polypyrrole chains to be much closer to one another. However, this is counterbalanced, since the number of chains is also greatly reduced by the space-restricting conditions present within the pores, which seriously limit inter-chain transport and thus limit the overall conductivity.

The dc conductivities of such composites are not greatly affected by the gradual increase in temperature. In our view, the slight increase or decrease in dc conductivity, which is still observed in the majority of the sample is mainly the result of the presence of polymeric material on the external surface of the host. This fraction of polymeric material will behave as conventional polypyrroles, as will be seen later for the analogous composites prepared by direct threading. By comparison with conventional polypyrroles, the rate of dc conductivity decay in polypyrroles intercalated in MCM-41 lattices is found to be much slower. Indeed, the dc conductivity of conventional polypyrroles was found, in average, to drop rapidly at $80^{\circ} \mathrm{C}$ in air. PPy(MBSA) exhibited drops up to two orders of magnitude over 6 months, PPy(DEHS) exhibited drops of one order of magnitude, and finally, the dc conductivity of PPy(DBSA) was found to decrease by two orders of magnitude over the same period of time. By comparison, PPy-MCM- $41^{\text {a }}$ composites prepared using similar synthetic procedures, exhibited average decreases of less than one order of magnitude, irrespective of the dopants present. If we believe that the small fraction of polymeric material present on the surface of the composites behave like conventional polypyrroles and degrade quite rapidly, we assume that the majority of the polymeric material is located within the silica lattices, and is relatively well protected from the outside atmospheric conditions, and degrades much more slowly. This limited contact between the polymeric material and the atmospheric oxygen or moisture results in a dramatic slow-down of the rate of dc conductivity decay. When retrieved by lattice dissolution or de-threading, polymeric materials exhibited similar behaviour to conventional polypyrroles, with a loss of dc conductivity by one or two orders of magnitude over 6 months.

\begin{tabular}{|l|c|c|c|c|c|c|}
\hline & DEHS & DBSA $_{\text {ex }}$ & MBSA & DBSA & Chloride & None \\
\hline $\begin{array}{l}\text { Direct threading } \\
\begin{array}{l}\text { In situ } \\
\text { polymerisation }\end{array}\end{array}$ & $10^{-4}-10^{-2}$ & $10^{-5}-10^{-4}$ & --- & --- & --- & --- \\
$10^{-6}$ & $10^{-7}-10^{-6}$ & $10^{-4}-10^{-3}$ & $10^{-8}-10^{-6}$ & $10^{-8}-10^{-7}$ & Insulator \\
\hline
\end{tabular}

Table 6: DC conductivities, $\sigma\left({\left.\mathrm{S} . \mathrm{cm}^{-1}\right)}^{-1}\right.$ of PPy-MCM-41 ${ }^{\mathrm{b}}$ composites

\subsection{Polypyrrole-silica composites produced by direct threading}

Direct threading of polypyrroles into the lattices of MCM-41 resulted in composites exhibiting higher polymer loading than that of analogues produced through in situ polymerisation. However, our beliefs are that the majority of this polymeric material is located near or on the external surfaces of the host material, rather than on

\footnotetext{
${ }^{\text {a }}$ Silylated or not.
} 
its internal surfaces. Indeed, infrared studies of these materials show features that are very similar to those observed in conventional polypyrroles. Such features do not correspond to the idea of composites where polypyrrole is homogeneously located throughout the host, especially when one takes into account that these composites are $80 \%$ silicate, and their composition should be reflected in their infrared spectra, as seen earlier for the analogues produced by in situ polymerisation. By contrast, such features will be consistent with composites where polypyrrole act as a coating rather than a guest. Whilst the hydrophilic environment present in MCM-41 is certainly a limiting factor in direct threading, similar procedures attempted in TMS-MCM-41 result in composites with similar or inferior polymeric loadings. This can only be explained by the fact that direct threading of linear polymeric chains in highly ordered silica nanopore systems seems to be partially affected by an associated loss of entropy, which in the case of polypyrroles produced by in situ polymerisation, is believed to be distributed over the individual steps of the propagation and then compensated for by the reaction energy liberated in each addition step.

These composites exhibit much higher dc conductivities than their analogues produced by in situ polymerisation. However, as seen earlier, the polymeric materials are not homogeneously distributed throughout the lattices, but rather concentrated on the external surfaces of the host, which leads us to believe that the recorded dc conductivity does not reflect the conductivity of the composite itself, but rather that of its polymeric coating, and this is further confirmed by the decrease or increase in dc conductivity as the temperature is increased, which mirrors the patterns observed for conventional polypyrroles. Thermal and environmental stability measurements show that PPy-MCM- $41^{\mathrm{a}}$ composites produced by direct threading are found to mirror those of conventional polypyrroles, with dc conductivities dropping rapidly at $80^{\circ} \mathrm{C}$ in air. These results further confirm our hypothesis that the direct threading procedures result in the deposition of a layer of polymeric material on the surface of the host, rather than in an adsorption of polymeric chains within the lattices of the host.

\section{CONCLUSION}

Nanoporous silicas with 3-D hexagonal structures were synthesised using methods relying on surfactant micelles as templates for the assembly and subsequent polymerisation of inorganic precursors at the surfactantsolution interface. The nanostructures of these silica materials were controlled by varying the synthetic conditions, such as chemical composition (surfactant/silica precursor ratio) and surfactant molecular structures. The subsequent APS and TMS silylations were found to be highly successful, with maximum degrees of silylation reaching values up to $30 \%$ and $20 \%$, for APS and TMS respectively.

Despite yielding composites exhibiting dc conductivities up to $10^{-2} \mathrm{~S}_{\mathrm{cm}} \mathrm{cm}^{-1}$, direct threading was found to be ineffective and yielded inhomogeneous composites, with polypyrrole-rich external surfaces. By contrast, in situ polymerisation of pyrrole monomer within the lattices of MCM-41 ${ }^{\mathrm{b}}$ resulted in PPy-MCM- $41^{\mathrm{b}}$ composites with polypyrrole-rich external and internal surfaces. When compared to PPy-Si composites produced with other silicates, PPy-MCM- $41^{\mathrm{b}}$ composites exhibited improved dc conductivity and environmental stabilities, and semiconducting properties. ${ }^{(19)}$ This improvement reached three orders of magnitude (up to $10^{-3} \mathrm{~S} . \mathrm{cm}^{-1}$ ), demonstratingd that the functionalisation of the internal and external surfaces of the hosts prior to polymerisation, through APS or TMS silylation, enabled us to adjust the monomeric intake and, consequently, increase the polymeric loading of the resulting PPy-Si composites (up to 14\% and 8\%, w/w, for APS and TMS respectively). However, our studies of the confined geometry of MCM- $41^{\text {a }}$ lattices on the growth of quasi-linear polymer chains, such as polypyrrole chains, led us to conclude that, in all cases, the length of the chains was significantly reduced when compared to conventional polypyrrole analogues (152 units vs. 260-850 units). ${ }^{(18)}$

With respect to applications, these hybrid composites could be of interest, e.g. for various sensors which require composites with low electrical conductivities, although the powder form somewhat restricts their use. Hybrid thin silica films, both supported and unsupported, would have the ideal morphology for commercial

\footnotetext{
${ }^{a}$ Silylated or not.

${ }^{\mathrm{b}}$ Silylated.
} 
applications. However, our early attempts to produce such composites via in situ electrochemical polymerisation have resulted in inhomogeneous composites with poor polypyrrole loadings. Our assumption is that the orientation of the pores affects the growth of the polymeric chains; indeed in situ electrochemical polymerisation requires the pores to be perpendicular to the substrate (electrode), whereas SEM studies of their cross-section have found that the cylindrical nanopores tend to run parallel to the substrate, irrespective of the substrate and synthetic method. ${ }^{(20)}$

\section{REFERENCES}

(1) Sixou; B.; Travers, J.P.; Vautrin, M; Attias, A.J. Synthetic Metals; 84, (1997) 835-836

(2) Epron, F.; Henry, F.; Sagnes, O. Makromol. Chem., Makromol. Symp.; 35-36; (1990) 527

(3) Tieke, B.; Gabriel, W. Polymer; 31; (1990) 20

(4) Street, G.B.; Lindsey, S.E.; Nazzal, A.I.; Wynne, K.J. Mol. Cryst. Liq. Cryst.; 118, (1985) 137

(5) De Paoli, M.A., Waltman, R.J.; Diaz, A.F.; Bargon, J. J. Polym. Sc. Part A Polym. Chem.; 23;(1985) 1687

(6) Park, Y.H., Han, M.H. J. Appl. Polym. Sc.; 45; (1992) 1973

(7) Bein, T.; Enzel, P. J. Phys. Chem. Soc. Chem. Commun.; (1989) 1326-1327

(8) Bein, T.; Enzel, P. Synthetic Metals; 29; (1989) 163-168

(9) Bein, T.; Enzel, P.; Beuneu, F.; Zuppiroli, L. Adv. Chem.; 226; (1990) 433-449

(10) Hill, P.G.; Foot, P.J.S., Budd, D.; Davis, R. Materials Science Forum; 122; (1993) 185-194

(11) Hill, P.G.; Foot, P.J.S., Budd, D.; Davis, R. Synthetic Metals; 76; (1996) 289-293

(12) Stucky, G.D.; Dwyer, F.G. Intrazeolite Chemistry; ACS Symp. Series, 218; Washington D.C. (1983)

(13) Kresge, C.T.; Leonowicz, M.E.; Roth, W.J.: Vartuli, J.C.; Beck, J.S. Nature; 359; (1992) 710

(14)) Attard, G.S.; Edgar, M.; Göltner, C.G. Acta Materialia; 46(3); (1998) 751

(15) Wielder; H.H. Laboratory Notes on Electrical and Galvanomagnetic Measurements, Material Science Monographs, 2, Elsevier, Amsterdam (1972)

(16) Cohan, L.H. J. Am. Chem. Soc.; 60; (1938) 433

(17) Perruchot, C.; Chehimi, M.M.; Delamar, M.; Eccles, J.A.; Steele, T.A.; Mair, D. Synthetic Metals; 113; (2000) 53-63

(18) Oh, E.J.; Jang, K.S.; MacDiarmid, A.G. Synthetic Metals; 65; (2002) 125

(19) Bein, T.; Enzel, P. Mol. Cryst. Liq. Cryst.; 181; (1990) 315-324

(20) Aksay, I.; Trau, M.; Manne, S.; Honma, I. Science, 273; (1996) 892 

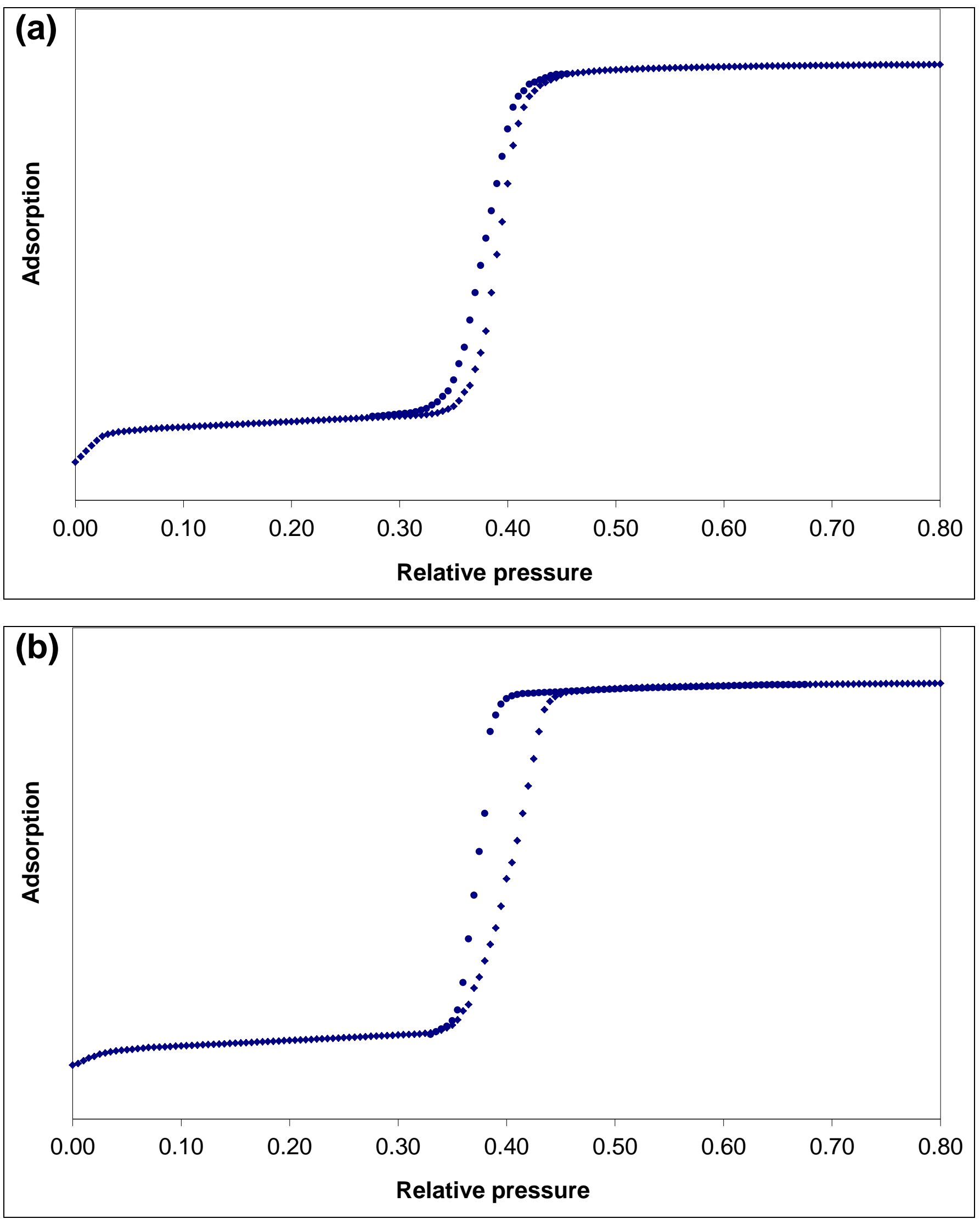

Figure 1: Nitrogen adsorption isotherms in (a) MCM-41, (b) APS-MCM-41 materials 

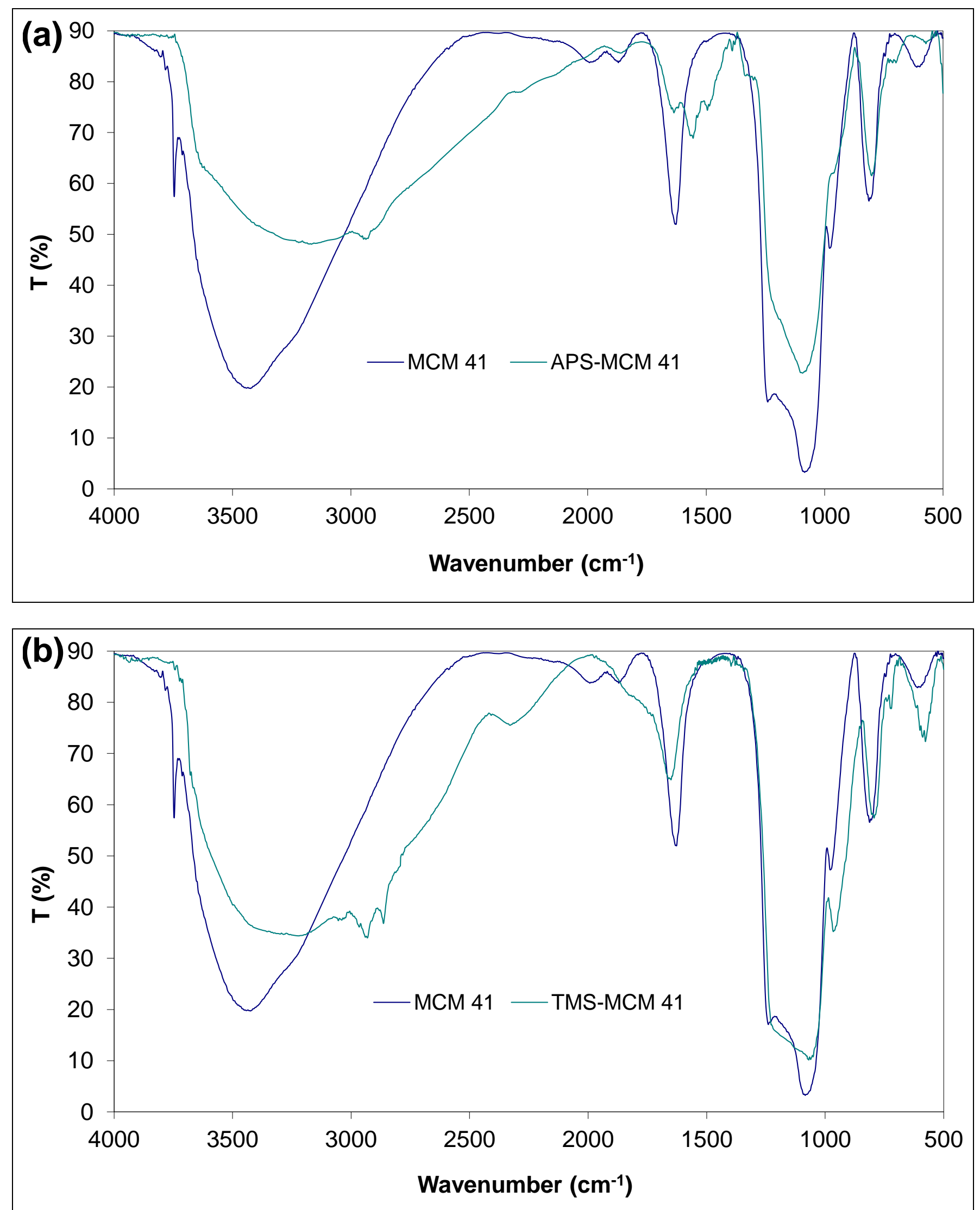

Figure 2: Diffuse reflectance FTIR spectra of MCM-41/APS-MCM-41, MCM-41/TMS-MCM-41 materials. 


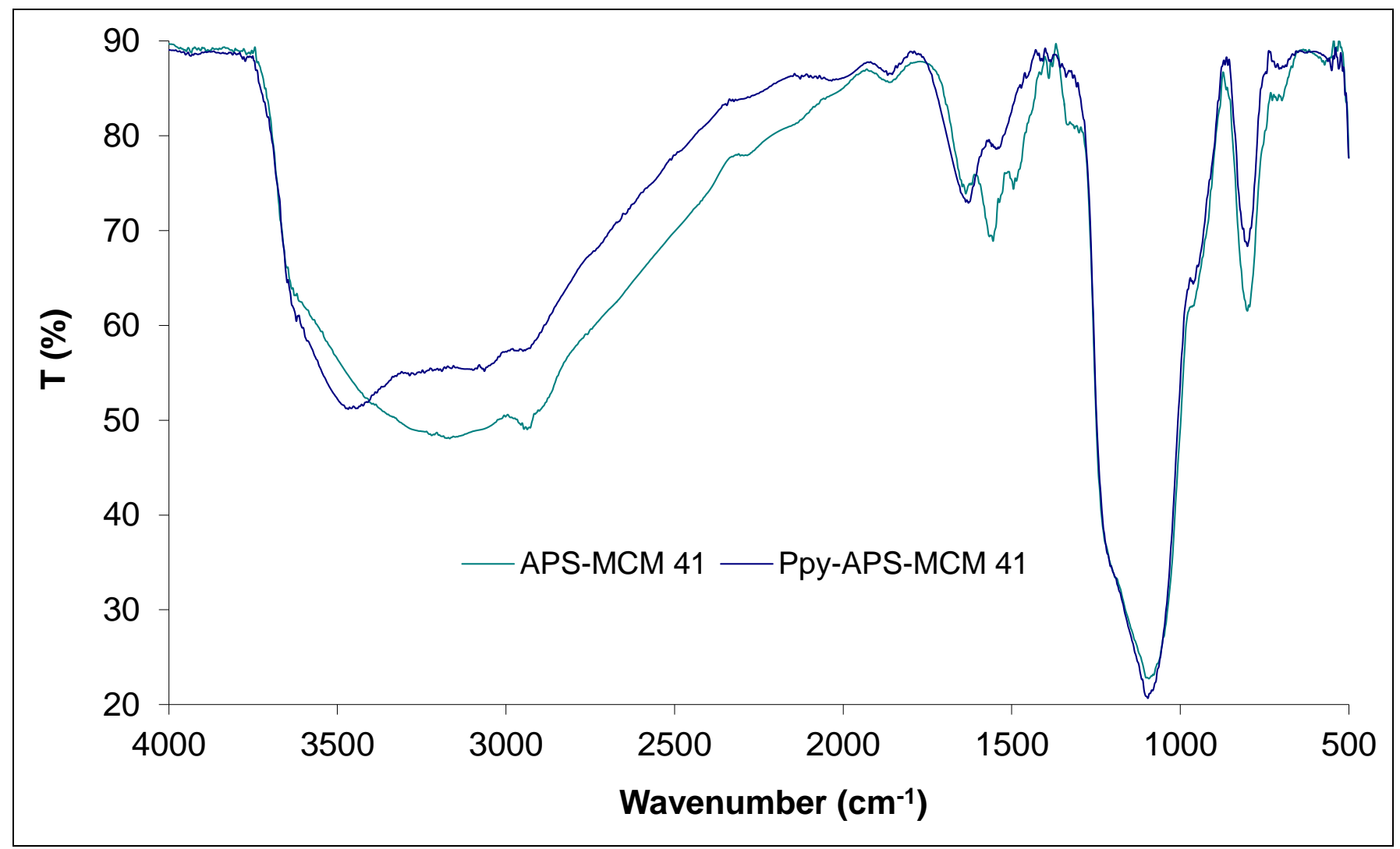

Figure 3: Diffuse reflectance FTIR spectra of Ppy-APS-MCM41 composites. 